\title{
Liposucción asistidla por ultrasonido para tratamiento de Ia ginecomastia: experiencia en los últimos 5 años
}

Ultrasound-assisted liposuction in gynecomastia management: last5-year experience

\author{
Ernesto A. MORETTI*, David CHANCHI**, \\ Jonathan CAMARGO**, Darío ALARCÓN ${ }^{* *}$
}

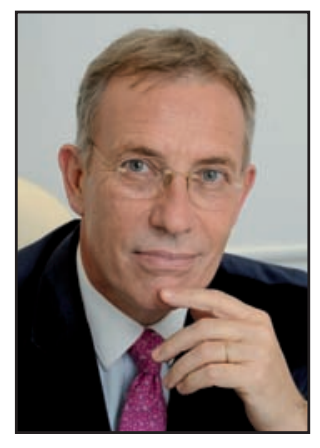

Moretti, E.A.
Resumen

Antecedentes y Objetivo. La liposucción asistida por ultrasonido quirúrgico (USAL por sus siglas en inglés) se utiliza ampliamente en el tratamiento de las lipodistrofias corporales. En la ginecomastia, está demostrado que la combinación de ultrasonido y liposucción presenta mayor efectividad en el resultado final.

Queremos dar a conocer la experiencia de nuestro Servicio en el abordaje de esta patología implementando la USAL como tratamiento único y como medida terapéutica coadyuvante de la cirugía abierta.

Material y Método. Estudio descriptivo, de cohorte única, durante el segundo semestre del 2017 con registros de todos los pacientes tratados por el Servicio de Cirugía Plástica del Sanatorio Los Arroyos y Hospital Privado de Rosario (Rosario, Argentina) entre 2011-2016. En total, 43 pacientes con edades comprendidas entre 16 y 70 años evaluados retrospectivamente y estratificados según el método de corrección quirúrgica en 2 grupos. Grupo 1 (n=26) tratamiento exclusivo con USAL y grupo $2(\mathrm{n}=17)$ USAL combinada con resección abierta de tejido glandular mamario.

Analizamos el tiempo de recuperación postquirúrgica, las complicaciones y la conformidad subjetiva de los pacientes.

Resultados. Ninguno de los casos presentó complicaciones que requirieran intervención adicional. Como complicación menor hubo 4 casos de equímosis torácica importante ( 2 en cada grupo). Todos presentaron adecuada retracción cutánea y conformidad con el resultado a los 30 días. Comparativamente, encontramos amplia diferencia en el tiempo de reintegro a las actividades cotidianas tras la intervención: media de 7 días para el grupo 1 frente a 11 días para el grupo 2 .

Conclusiones. La USAL es una importante herramienta terapéutica en ginecomastia, de especial relevancia en casos de predominio graso dado que por si sola es un tratamiento efectivo en estos casos. En los casos de ginecomastia mixta que requieren resección quirúrgica, la USAL es una importante medida coadyuvante para un resultado postquirúrgico óptimo con adecuada retracción cutánea y mínimas complicaciones.

\section{Palabras clave Ginecomastia, Liposucción, Ultrasonido.}

Nivel de evidencia científica

Recibido [esta versión]

Aceptado
$4 c$ Terapéutico

23 febrero/2018

$15 \mathrm{marzo} / 2018$

\begin{abstract}
Background and Objective. Surgical Ultrasound assisted liposuction (USAL) is widely used in the management of body lipodystrophies. In gynecomastia, it has been reported that thus combination of ultrasound and liposuction has proven an improved effectiveness and better outcomes. We present our experience in the management of this pathology using USAL exclusively and as adjuvant measure of conventional open surgery.
\end{abstract}

Methods. A descriptive and single cohort study was performed during the second semester of 2017 with patients' records from the Plastic Surgery Department of Sanatorio Los Arroyos and Hospital Privado de Rosario (Rosario, Argentina), treated between 2011-2016. A total of 43 patients diagnosed with gynecomastia, with ages between 16 and 70 years, retrospectively assessed and stratified into 2 groups according the therapeutical approach. Group 1, treated with USAL exclusively $(n=26)$ and group 2 , treated with combined USAL and open mammary glandular tissue excision surgery $(n=17)$.

Postoperative downtime, complications and patient's satisfaction were assessed.

Results. None had any major complications requiring additional measures. The only reported minor complication was significant thoracic bruising in 4 patients ( 2 in each group). All patients showed adequate skin retraction and 30 days post-operative results satisfaction. We found wide differences when compared the reported postoperative downtime, where group 1 reported a mean of 7 downtime days versus 11 downtime days for group 2 .

Conclusions. USAL is an important therapeutical measure when treating patients diagnosed with gynecomastia. It's especially useful when treating gynecomastia with fatty dominance, being effective by itself. Additionally, when treating patients with significant glandular tissue which needs surgical excision (mixed gynecomastia), USAL seems to be an useful adjuvant measure to achieve an outstanding outcome with optimal skin retraction and minimal complication rates.

\begin{tabular}{|lr|}
\hline Key words & $\begin{array}{l}\text { Gynecomastia, Liposuction, } \\
\text { Ultrasound. }\end{array}$ \\
Level of evidence & $4 \mathrm{c}$ Therapeutic \\
Received lthis version] & $23 \mathrm{february} / 2018$ \\
Accepted & $15 \mathrm{march} / 2018$ \\
\hline
\end{tabular}

Conflicto de intereses: los autores declaran no tener ningún interés financiero relacionado con el contenido de este artículo

\footnotetext{
* Jefe de Servicio

** Médico Residente

Servicio de Cirugía Plástica, Hospital Privado Rosario, acreditado por la Sociedad Argentina de Cirugía Plástica.
} 


\section{Introducción}

La ginecomastia consiste en el aumento exagerado de tamaño de las mamas en el varón, descrita inicialmente por Paulos de Aegina (635 - 690 d. de C). ${ }^{(1)}$ Sabemos que entre un 32 y un $65 \%$ de la población masculina la padece $^{(3)}$ siendo más común en la séptima década de la vida. ${ }^{(4)}$ Generalmente es asintomática, pero afecta considerablemente a la autoestima de los pacientes que la padecen, produciendo ansiedad, incomodidad social y vergüenza ${ }^{(5)}$ que suelen ser motivo de consulta médica. Habitualmente es de presentación bilateral; sin embargo en los casos de presentación unilateral el enfoque diagnóstico debe basarse en descartar un proceso maligno.

Puede presentarse por causa hormonal desde el nacimiento (paso de hormonas maternas al feto), en la pubertad o en la edad adulta, produciéndose de manera fisiológica por un desequilibrio entre testosterona - estrógenos en el tejido mamario masculino. ${ }^{(6)}$ También puede encontrarse en patologías como la cirrosis hepática, la desnutrición, el hipogonadismo, el síndrome de Klinefelter, neoplasias, enfermedades renales, hiper o hipotiroidismo. ${ }^{(7)}$

$\mathrm{Su}$ presentación secundaria a efectos medicamentosos se ha asociado a diferentes fármacos de uso común como: estrógenos, digitálicos, ranitidina, espironolactona, metronidazol, finasteride, ketoconazol, cimetidina, diazepam entre otros y al consumo de marihuana y etanol. ${ }^{(8)}$

La ginecomastia puede ceder espontáneamente, como sucede en el neonato y en la pubertad, pero en muchas ocasiones requiere tratamiento, ya sea farmacológico o quirúrgico.

La clasificación más utilizada es la descrita por Simon en 1973, según el tamaño y la redundancia de piel (Tabla I).(2) Se considera que el tratamiento con técnicas de lipoaspiración está indicado para los grados I y IIa y la resección mixta para los grados mayores.

De igual forma, están descritas diferentes técnicas y procedimientos quirúrgicos con diferentes cicatrices resultantes según el abordaje, como son: A) Puertos axilares; en el caso de realizar lipusucción o resección con shaver. P) Periareolares inferiores; si hay resección del componente glandular y no existe redundancia de piel. C) Cicatrices en surco submamario (horizontales); cuando se realiza pexia del complejo areola-pezón resecando piel y tejido redundante. D) Resultante en $\mathrm{T}$ invertida (en patrón de Wise); si el volumen mamario es

Tabla I. Clasificación de Simon de la ginecomastia(2)

Grado I . Pequeño aumento de volumen mamario visible, sin piel redundante.

Grado II A. Moderado aumento de volumen mamario, sin piel redundante.

Grado II B. Moderado aumento de volumen mamario, con piel redundante.

Grado III. Severo aumento de volumen mamario, con piel redundante (como una mama ptósica femenina). mucho mayor, como en el caso de pacientes postbariatricos. E) Cicatriz círcumareolar sumada a cicatriz vertical o en J, que se puede extender hasta región dorsal si la resección es mayor. ${ }^{(3)}$

El ultrasonido quirúrgico es una herramienta útil como pretratamiento para extraer grasa en pacientes con lipodistrofias, ya sean generales o localizadas; disminuye a su vez la aspiración de sangre ya que por su frecuencia de 2-3 MHz es selectivo para los adipocitos. Las células de tejido graso son desalojadas de sus tejidos de soporte por el efecto de transmisión acústica generado por las sondas, las cuales no afectan a tejidos más densos y por tanto respeta los componentes nerviosos, sanguíneos y las fibras de colágeno. ${ }^{(4)}$

Presentamos una investigación retrospectiva de pacientes intervenidos por ginecomastia bilateral entre los que separamos 2 grupos según el componente predominante del tejido mamario, y por tanto el tratamiento quirúrgico empleado, siendo en ambos casos ayudado por liposucción asistida por ultrasonido (USAL por las siglas del inglés ultrasound assisted liposuction). Este estudio se enfoca en mostrar nuestra experiencia en la utilización del ultrasonido tanto en liposucción como en procedimientos mixtos con resección abierta, evaluar nuestra incidencia de complicaciones asociadas al procedimiento y comparar los tiempos de recuperación postoperatoria en ambos grupos.

\section{Material y método}

Incluimos en nuestro estudio 43 pacientes de edades comprendidas entre los 14 y 70 años (media de 41 años) con ginecomastia bilateral, que consultaron en el Servicio de Cirugía Plástica del Sanatorio Los Arroyos y Hospital Privado Rosario (Grupo Gamma de Salud, Rosario, Argentina) entre los años 2011 a 2016. A la totalidad de los pacientes se les explicó de forma previa el procedimiento quirúrgico a emplear, sus implicaciones, posibles complicaciones y posteriormente, se procedió a la autorización del mismo mediante la firma de un consentimiento informado. En el caso de los menores de edad la autorización fue dada por sus tutores legales. La totalidad de los pacientes aceptaron el procedimiento.

A todos los pacientes se les solicitó ecografía mamaria bilateral prequirúrgica que informó sobre el exceso de tejido mamario a expensas del tejido graso (Grupo 1) (Fig. 1) o de tejido mixto: glandular y adiposo (Grupo 2) (Fig. 2) confirmando el diagnóstico de ginecomastia. Excluimos del estudio a los pacientes con ginecomastia primaria sin aumento del tejido adiposo (ginecomastia glandular pura primaria). En las ecografías practicadas se comprobó que no había presencia de masas mamarias en ninguno de los casos, descartándose también en el examen físico. Dos de los pacientes presentaban antecedente de tumor prostático y se encontraban en tratamiento farmacológico (ginecomastia debida a medicación con an- 

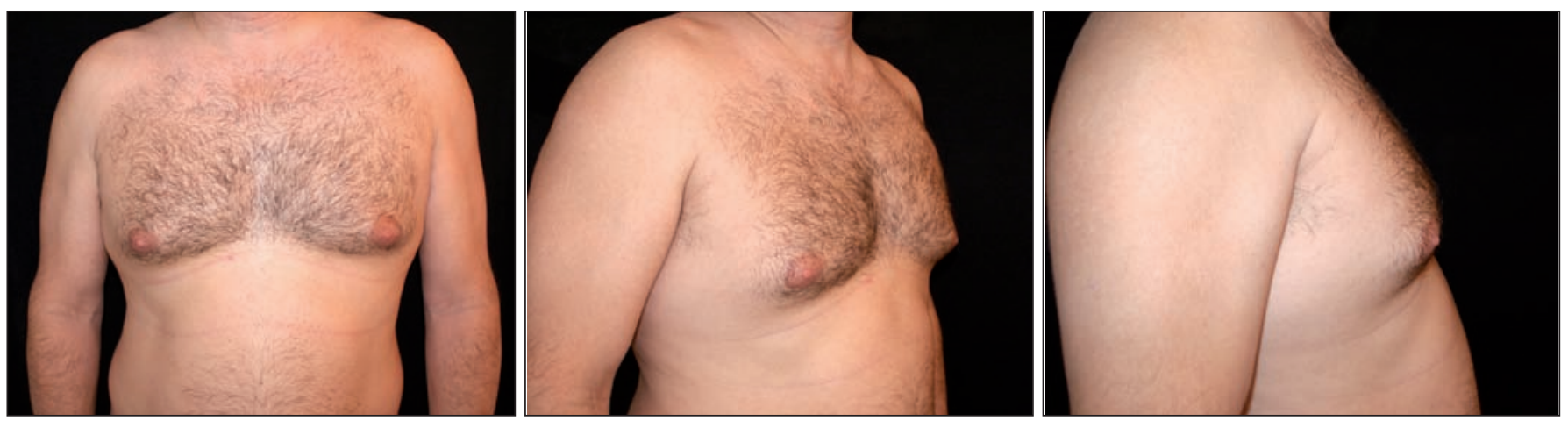

Fig. 1. Ginecomastia típica con aumento a expensas del tejido adiposo. Grupo 1: Indicación de liposucción asistida con ultrasonido (USAL).
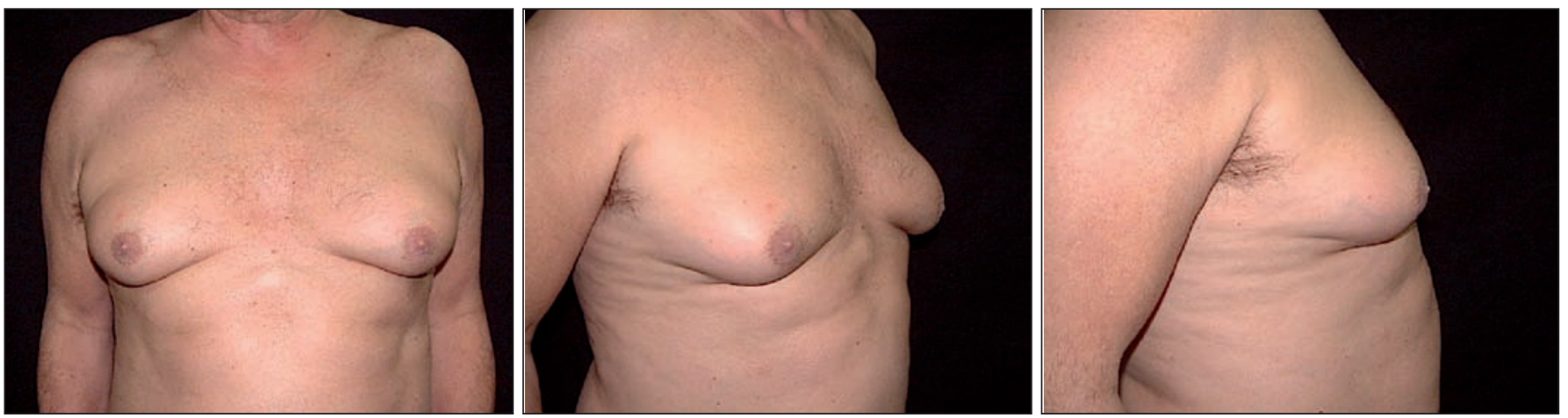

Fig. 2. Ginecomastia típica con aumento a expensas del tejido glandular mamario y adiposo. Gupo 2: Indicación de resección directa de la glándula mamaria y liposucción asistida con ultrasonido (USAL).

Tabla II. Estratificación de los pacientes del grupo de estudio

\begin{tabular}{|l|c|c|}
\hline & Grupo 1 & Grupo 2 \\
\hline $\mathbf{N}^{\mathbf{0}}$ de pacientes & 26 & 17 \\
\hline Edad promedio (años) & $15-59(36)$ & $14-70(36)$ \\
\hline SEVERIDAD* & & \\
\hline Grado I & 5 & 7 \\
\hline Grado IIA & 4 & 4 \\
\hline Grado IIB & 11 & 3 \\
\hline Grado III & 6 & 3 \\
\hline
\end{tabular}

*Según clasificación de Simon para ginecomastia

tiandrógenos). Adicionalmente, 2 de los pacientes ya habían sido previamente intervenidos, de forma extrainstitucional, para corrección de ginecomastia y presentaban disconformidad con el resultado obtenido, motivo por el cual solicitaron valoración y reintervención.

De los 43 pacientes, 13 eran menores de edad (ginecomastia primaria); 25 casos presentaban flacidez cutánea y 1 solo paciente presentó asimetría mamaria importante (Tabla II).

Estratificamos a los pacientes en 2 grupos según el componente predominante de su tejido mamario según la valoración ecográfica: 26 casos de aumento del componente adiposo (incluidos en el grupo 1) y 17 casos de aumento del componente glandular y del compartimiento adiposo (incluidos en el grupo 2). Realizamos USAL de forma exclusiva y USAL más resección abierta (técnica mixta) para cada grupo respectivamente.

En todos los casos, realizamos la marcación prequirúrgica con el paciente en bipedestación, delimitando el área pectoral tanto en la porción superior como en la lateral, definiendo así los límites de la liposucción. Marcamos también los puertos de entrada para las cánulas sobre la línea axilar anterior de forma bilateral, y el puerto de entrada a nivel axilar (Fig. 3). En 15 de los 17 pacientes del grupo 2 (a quienes se les practicó USAL mas cirugía abierta), realizamos marcación periareolar inferior para abordaje mínimo de resección abierta de glándula mamaria. Los 2 casos restantes correspondieron a los pacientes con ginecomastia severa por tratamiento con antiandrógenos, a quienes les realizamos marcación para abordaje por surco submamario con la finalidad de lograr acceso completo al tejido glandular.

Llevamos a cabo todos los procedimientos bajo anestesia general con infiltración de solución tumescente previa a la incisión, usando cánulas romas (tipo Klein) y jeringas de $60 \mathrm{cc}$. La mezcla utilizada para la solución fue de $500 \mathrm{cc}$ de suero salino al $0.9 \%$, más $20 \mathrm{cc}$ de lidocaína al $2 \%$ con epinefrina 1:1.000.000, más $1 \mathrm{mg}$ adicional de adrenalina (modificación de la solución tumescente de Klein) ${ }^{(5)}$ La incisión en los puertos marcados la hicimos de $2 \mathrm{~mm}$ con hoja de bisturí numero 11, e inyectamos entre 400 y $500 \mathrm{cc}$ de solución tumescente en cada mama. A los pacientes en los que practicamos abordaje abierto, les hicimos la infiltración de la incisión con jeringa de 20 cc y aguja intramuscular $21 \mathrm{G}$ directamente a nivel periareolar inferior y en todo el complejo areola-pezón. 
Procedimos a continuación al uso del ultrasonido quirúrgico con un equipo VASER ${ }^{\circledR}$ (Sound Surgical Technologies, Colorado, EE.UU.) entrando a través de los puertos marcados y empleando una frecuencia de $36.000 \mathrm{~Hz}$, con calibración de potencia al $70 \%$ y con sonda de titanio de 2 anillos de $3.7 \mathrm{~mm}$ (provistas por el fabricante). Previa colocación de medidas de protección cutánea (compresas húmedas estériles) realizamos barrido en forma de abanico durante 10 minutos en cada mama.

Posteriormente continuamos con la succión de los tejidos licuefactados, empleando los mismos puertos de in- greso, y mediante cánulas Vent $\mathrm{X}^{\circledR}$ (Sound Surgical Technologies, Colorado, EE.UU.) multifenestradas, de punta roma, de 2.7 y $3.5 \mathrm{~mm}$ de diámetro (Fig. 4 y 5), hasta obtener el tamaño deseado de la glándula mamaria según maniobra de pellizco (pinch test). Suturarmos los puertos con puntos simples de nylon 5-0 y aplicamos Adhesol ${ }^{\circledR}$ (resina de colofonia/esencia de trementina) (Instituto Biológico Argentino, Argentina) en la superficie cutánea adyacente al puerto; cubrimos con gasa estéril.

Para los pacientes del grupo 2, tras la realización de la USAL, llevamos a cabo incisión por abordaje periareolar
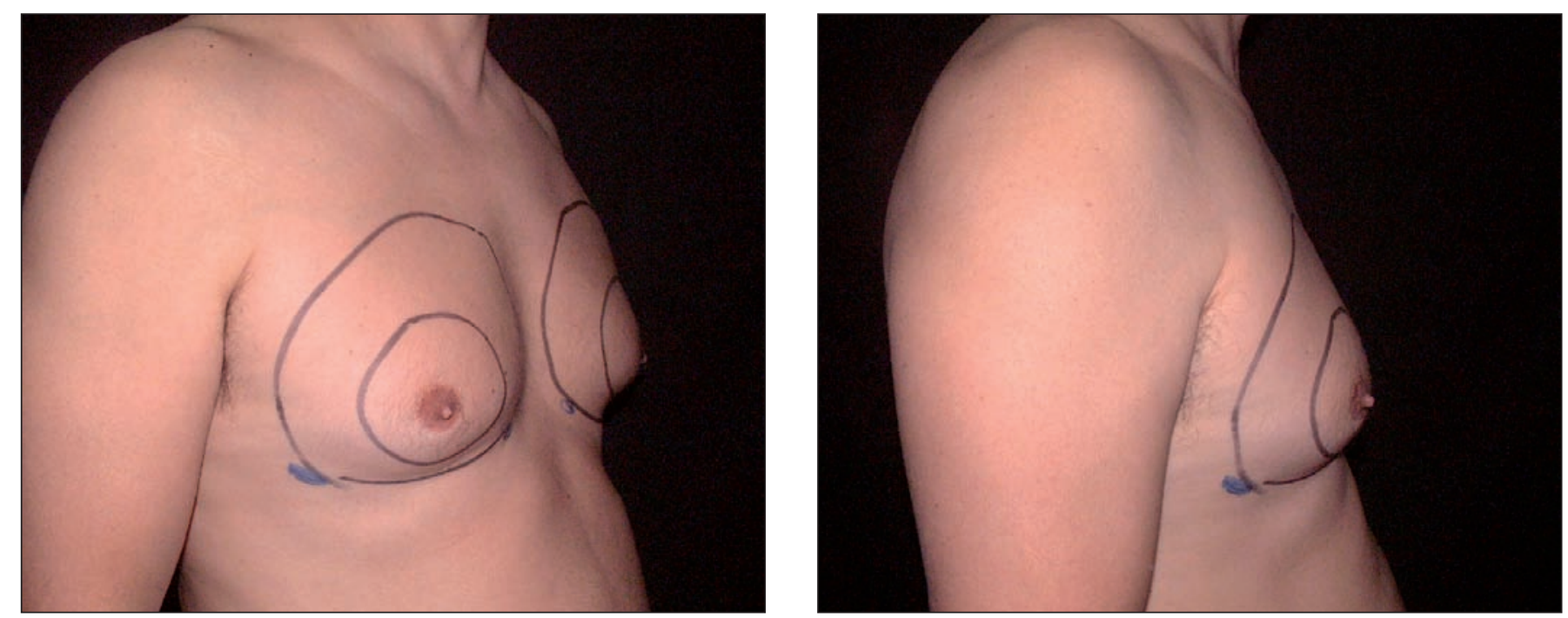

Fig. 3. Marcación de la zona de uso del ultrasonido y de los puertos de ingreso.

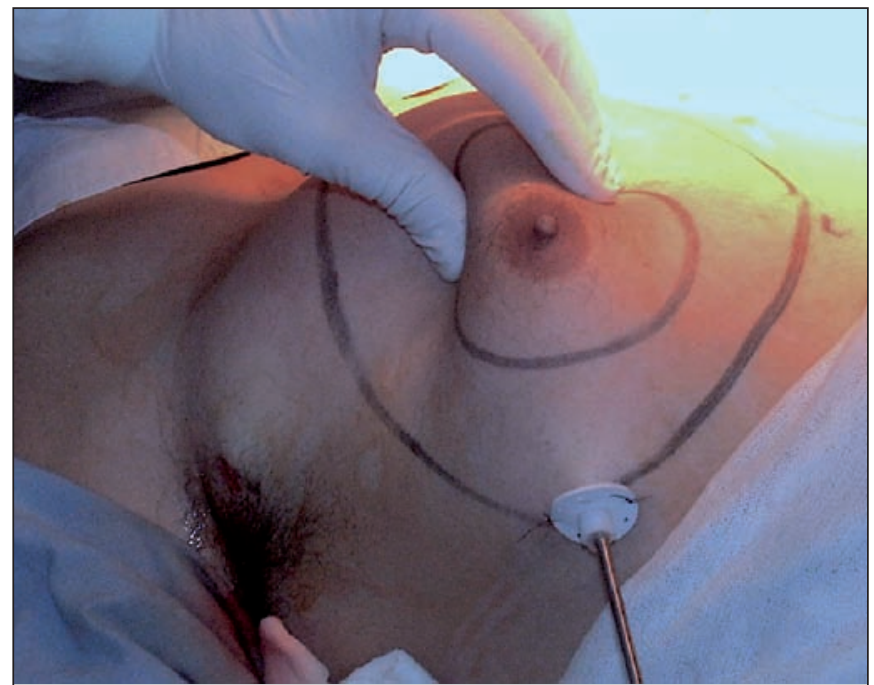

Fig. 4. Puertos de ingreso con protección al usar ultrasonido quirúrgico: sonda de titanio de 2 anillos, de $3.7 \mathrm{~mm}$.
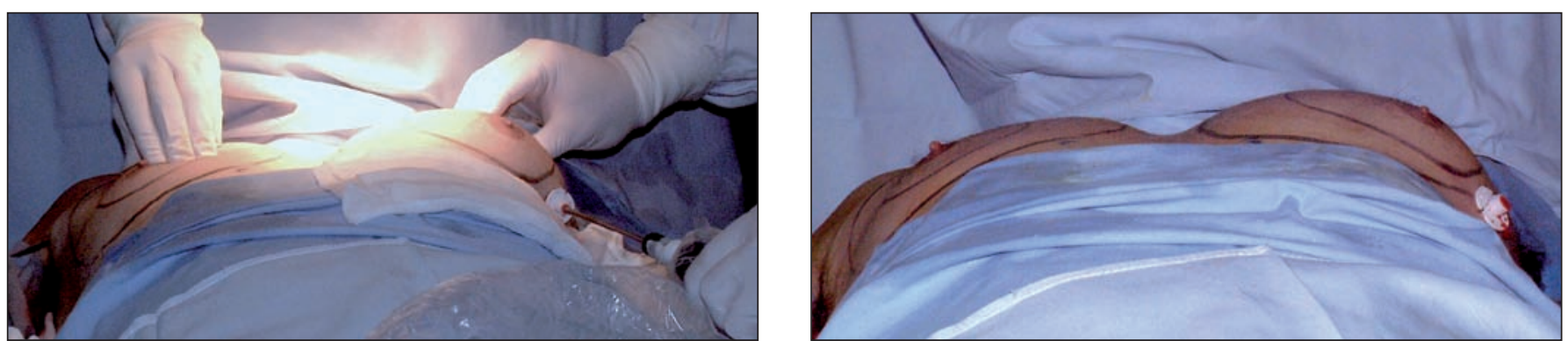

Fig. 5. Mama izquierda: uso de ultranido; mama derecha: succión tras el uso de ultrasonido, con cánulas multifenestradas de 3.5 mm. 
Tabla III. Cantidad de tejido extraído

\begin{tabular}{|l|c|c|}
\hline & Grupo 1 & Grupo 2 \\
\hline LIPOASPIRACIÓN (ml) & & \\
\hline Volumen máximo & 1100 & 850 \\
\hline Volumen mínimo & 150 & 100 \\
\hline Promedio lipoaspirado & 511.5 & 363.5 \\
\hline RESECCIÓN DE TEJIDO (gr) & & \\
\hline Resecado máximo & - & 80 \\
\hline Resecado mínimo & - & 15 \\
\hline Promedio resecado & - & 28.5 \\
\hline
\end{tabular}

Tabla IV. Tiempo de reintegro a las actividades cotidianas y satisfacción del paciente

\begin{tabular}{|l|c|c|}
\hline & Grupo 1 & Grupo 2 \\
\hline Dolor (Escala EVA) & & \\
\hline Mínimo y máximo reportados & $4-6$ & $5-7$ \\
\hline Promedio & 5 & 6 \\
\hline Satisfacción * & & \\
\hline Mínimo y máximo reportados & $3-5$ & $3-5$ \\
\hline Promedio & 4.7 & 4.5 \\
\hline Tiempo de reintegro (días) & & \\
\hline Mínimo y máximo reportado & $5-15$ & $6-15$ \\
\hline Promedio & 7 & 11 \\
\hline
\end{tabular}

EVA: Escala visual análoga

${ }^{\star}$ Escala validada por Krasopoulos y col.(6)

inferior, disección roma de la glándula mamaria y resección del tejido redundante con electrobisturí, teniendo especial precaución para preservar el botón mamario y evitar depresión del mismo en el postoperatorio. Suturamos la areola con puntos separados de nylon 5-0. A la totalidad de los pacientes les colocamos un vendaje compresivo con espuma de poliuretano y venda elástica durante 5 días hasta el primer control postoperatorio.

Los pacientes fueron dados de alta a las 8 horas de la cirugía y a todos se les recetó antibiótico profiláctico (cefadroxilo a dosis de $500 \mathrm{mg}$ cada 12 horas), analgésico antinflamatorio (diclofenaco/paracetamol, 50/300 mg cada 8 horas) e indicación de deambulación temprana.

En el postoperatorio, los pacientes fueron citados a los 5 días de la intervención para la primera cura; en ese momento retiramos el poliuretano e indicamos compresión con venda elástica continua durante 10 días más. Posteriormente fueron valorados a los 15, 30 y 60 días. Tomamos registro fotográfico previo al procedimiento y a los 2 meses, siendo en algunos casos el control fotográfico a los 6 meses y al año de postoperatorio.

\section{RESULTADOS}

En total recogimos 43 pacientes tratados por ginecomastia bilateral; 2 de ellos con ginecomastia secundaria a tratamiento farmacológico para cáncer prostático y los restantes sin causa determinada, considerándose estos como casos de ginecomastia fisiológica.

El total de grasa extraída en ambos grupos osciló entre los 100 y $1100 \mathrm{ml}$ de grasa (media de $500 \mathrm{ml}$ para el grupo 1 y de $360 \mathrm{ml}$ para el grupo 2). En el grupo $2 \mathrm{el} \mathrm{te-}$ jido mamario extraído pesó entre 10 y 45 gr. (media de 16 gr) (Ver Tabla III).

En 2 de los casos del grupo 1 se presentó en el postoperatorio equímosis considerable en ambos hemitórax (7.7\%); se estudió y descartó en ambos pacientes que hubiera trastornos de la coagulación. No se presentaron hematomas, colecciones ni seromas en el postoperatorio. Tampoco hubo casos de infección del sitio operatorio, signos de sufrimiento de piel, isquemia de región pectoral ni del complejo areola-pezón, así como tampoco pérdida de sensibilidad en los mismos.

En el postoperatorio mediato (día 5), valoramos la presencia de dolor mediante el uso de escala visual análoga (EVA) en la que 0 era inexistente y 10 el dolor máximo. Los pacientes a los que se les realizó procedimiento USAL exclusivo (grupo 1) lo describieron como tolerable (entre 4 y 5 ) y cedía con consumo de diclofenaco más paracetamol a la dosis indicada de 50/300 mg cada 8 horas. El grupo de pacientes de cirugía mixta (grupo 2) manifestó hiperalgesia sobre la herida quirúrgica en el reborde areolar (calificando el dolor entre 6 y 7 ) y requirieron añadir ketorolaco sublingual a dosis de $10 \mathrm{mg}$ de manera intermitente (Tabla IV).

El grupo 1 se reintegro a sus actividades cotidianas en un promedio de 7 días (entre 5 y 8 días) mientras que del grupo 2 lo hizo en un promedio de 11 días (entre 6 y 15 días).

En todos los casos hubo satisfacción con el resultado obtenido (media de 4.6/5 con valores registrados entre 3 y 5). Para efectos del estudio implementamos una modificación del cuestionario creado por Krasopoulos ${ }^{(6)}$ que permite evaluar el grado de satisfacción que tienen los pacientes con el procedimiento al cual fueron sometidos. Esta modificación consta de 15 preguntas que evalúan el impacto social, estético y físico que ha acarreado la cirugía. El cuestionario fue administrado vía telefónica usando los registros de la historia clínica al momento de realizar el estudio. Cuantificamos el grado de satisfacción de 1 a 5, siendo 1 muy insatisfecho y 5 extremadamente satisfecho.

Evidenciamos una notable retracción cutánea y del complejo areola-pezón además del aplanamiento del mismo en el 100\% de los pacientes; en 1 caso se presentó exceso de piel y ninguno requirió reintervención quirúrgica.

En el seguimiento a 1 año de los pacientes no encontramos casos de recidiva de la ginecomastia (Fig. 6-13). 

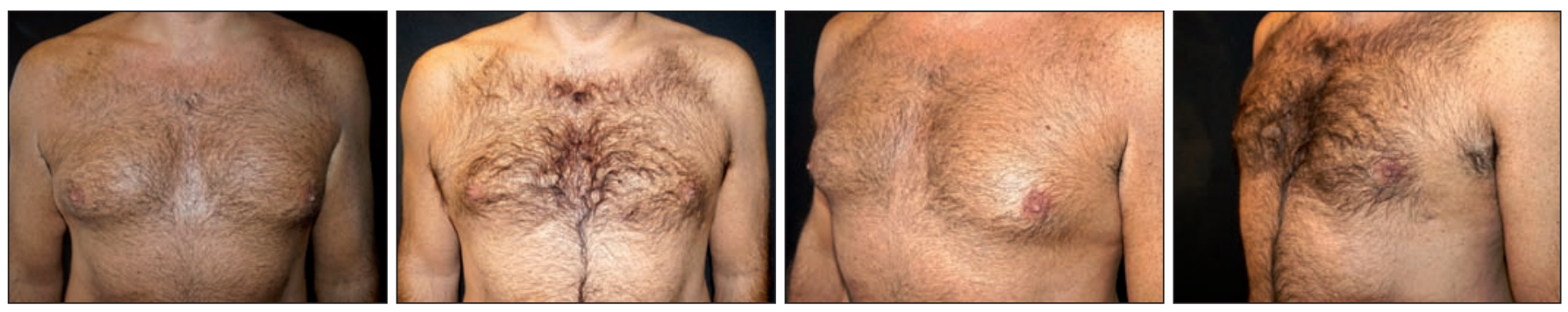

Fig. 6. Paciente de 36 años. Grupo 1 (Ginecomastia adiposa): resultado al año de postoperatorio. Liposucción asistida por ultrasonido exclusivamente.
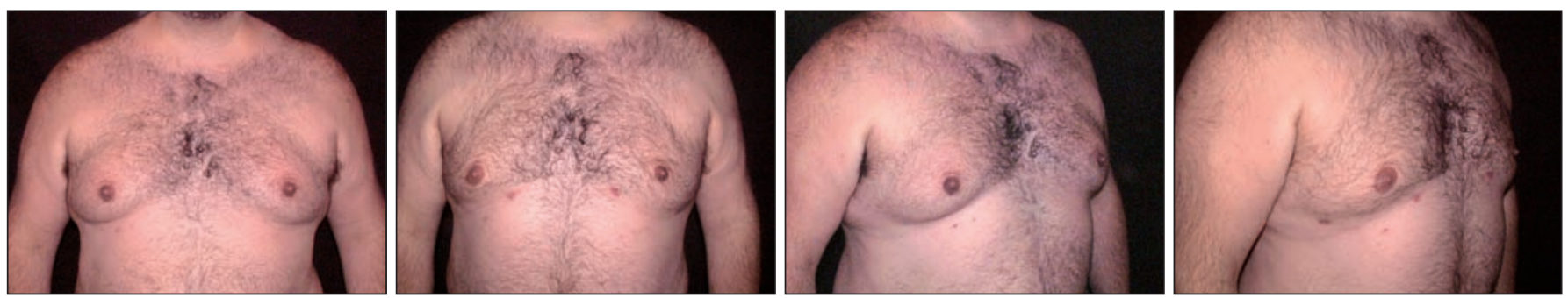

Fig. 7. Paciente de 56 años. Grupo 1 (Ginecomastia adiposa). Liposucción asistida por ultrasonido exclusivamente pre y postoperatorio a los 6 meses.
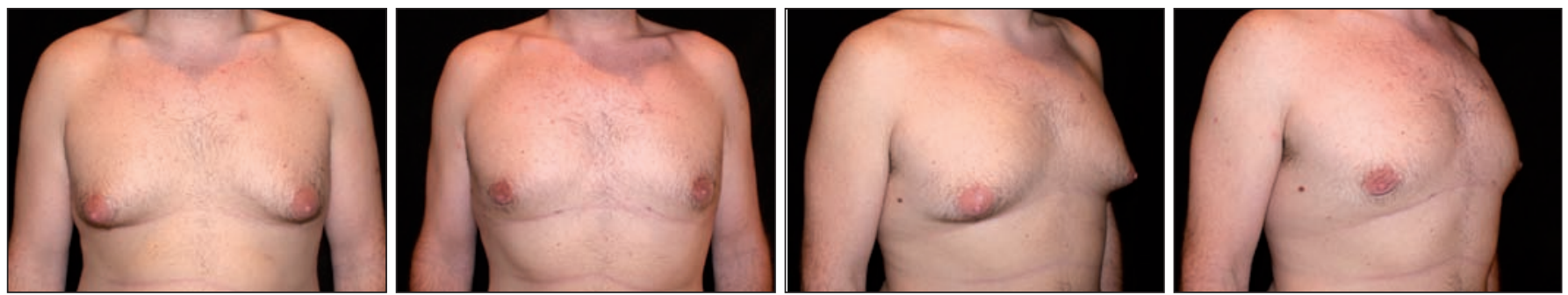

Fig. 8. Paciente de 31 años. Grupo 2 (Ginecomastia adiposa y glandular). Liposucción asistida por ultrasonido con retirada de glándulas por vía periareolar inferior. Pre y postoperatorio al año.
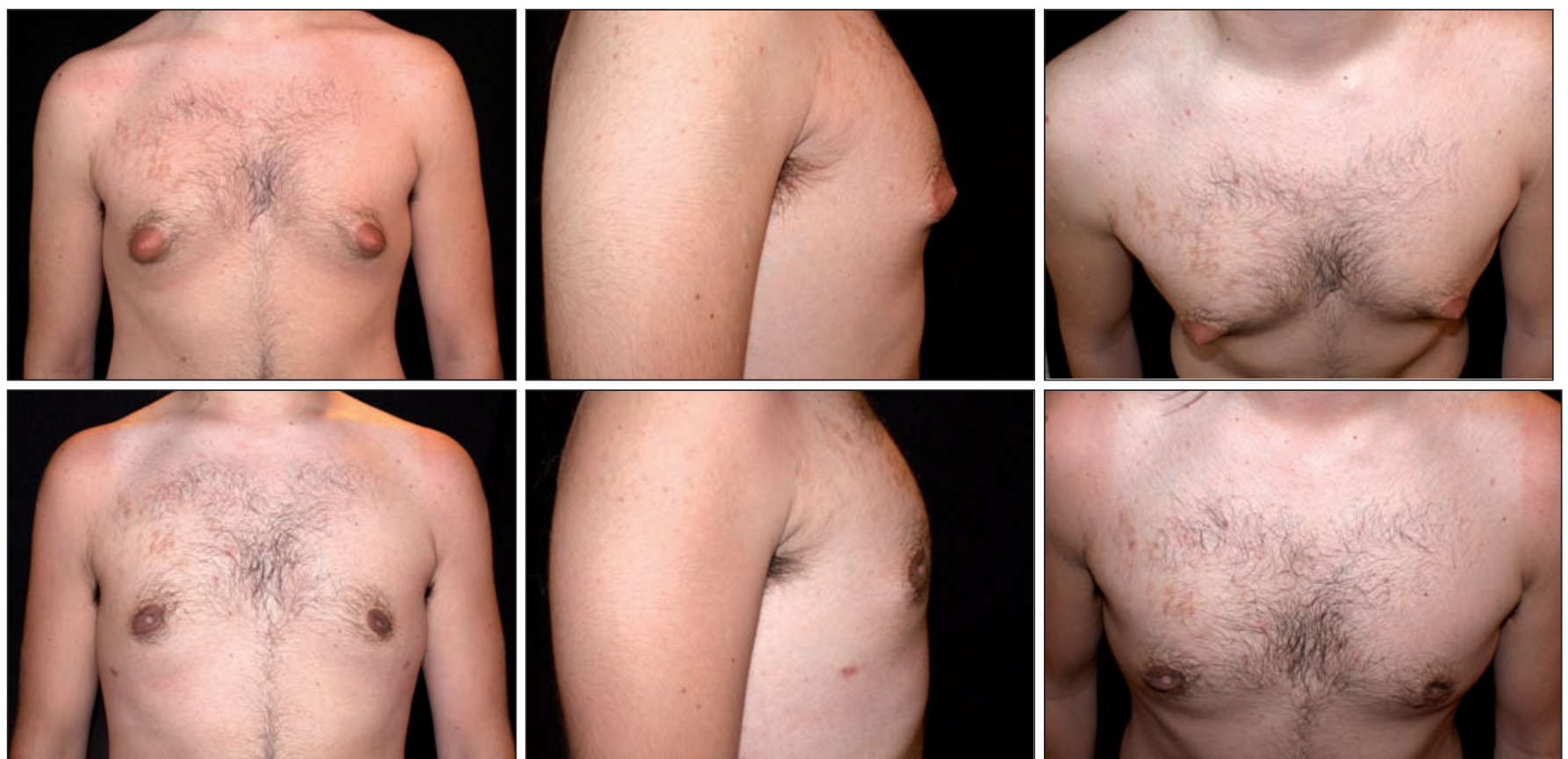

Fig. 9. Paciente de 29 años. Grupo 2 (Ginecomastia adiposa y glandular). Liposucción asistida por ultrasonido con retirada de glándulas por vía periareolar inferior. Resultado a los 6 meses de postoperatorio.

\section{Discusión}

Están descritos diferentes tipos de técnicas quirúrgicas para el tratamiento de la ginecomastia, desde cirugía abierta con distintas vías de abordaje ${ }^{(1)}$ hasta liposucción realizada con mínima incisión, asistida o no por láser, ul- trasonido o por shaver. Según Petty, ${ }^{(7)}$ todas las técnicas han demostrado efectividad en cuanto a la disminución en el tamaño de las mamas masculinas, unas con unas ventajas más evidentes que otras, como por ejemplo menor cicatriz resultante, menor dolor en el postoperatorio y mejor retracción de piel..$^{(8,9)}$ 

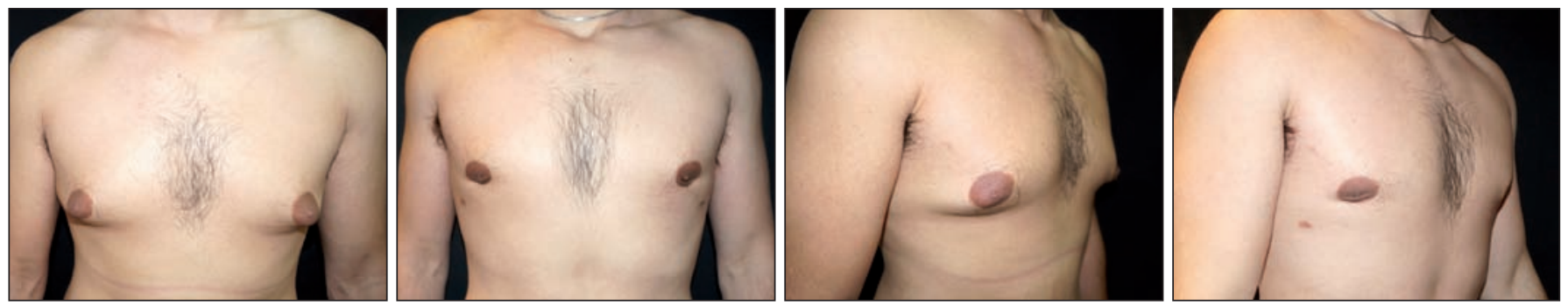

Fig. 10. Paciente de 24 años. Grupo 2 (Ginecomastia adiposa y glandular). Liposucción asistida por ultrasonido con retirada de glándulas por vía periareolar inferior. Pre y postoperatorias a los 2 meses.
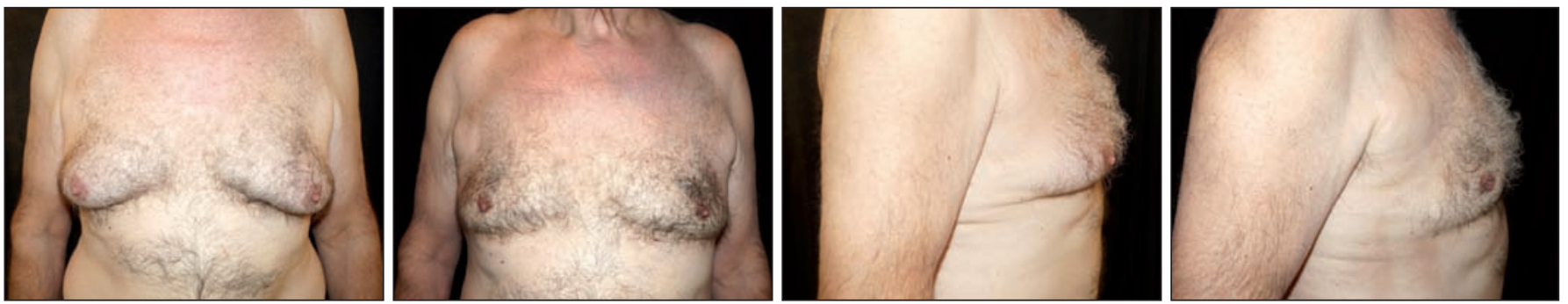

Fig. 11. Paciente de 68 años. Grupo 1 (Ginecomastia adiposa). Postoperatorio al año. Liposucción asistida por ultrasonido exclusivamente con retracción cutánea muy importante.
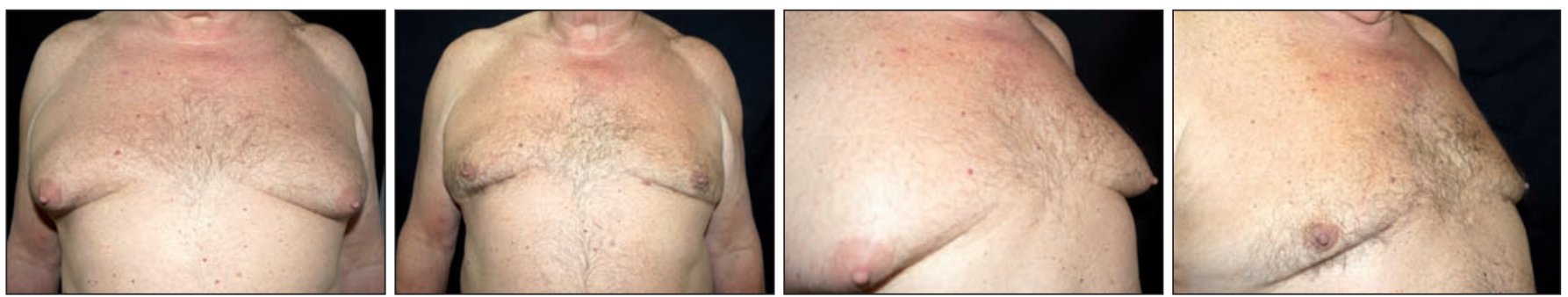

Fig. 12. Paciente de 68 años. Grupo 2 (Ginecomastia adiposa y glandular). Tratamiento con antiandrógenos por Ca. de próstata. Liposucción asistida por ultrasonido con retirada de glándulas vía surco submamario. Pre y Postoperatorio al año.
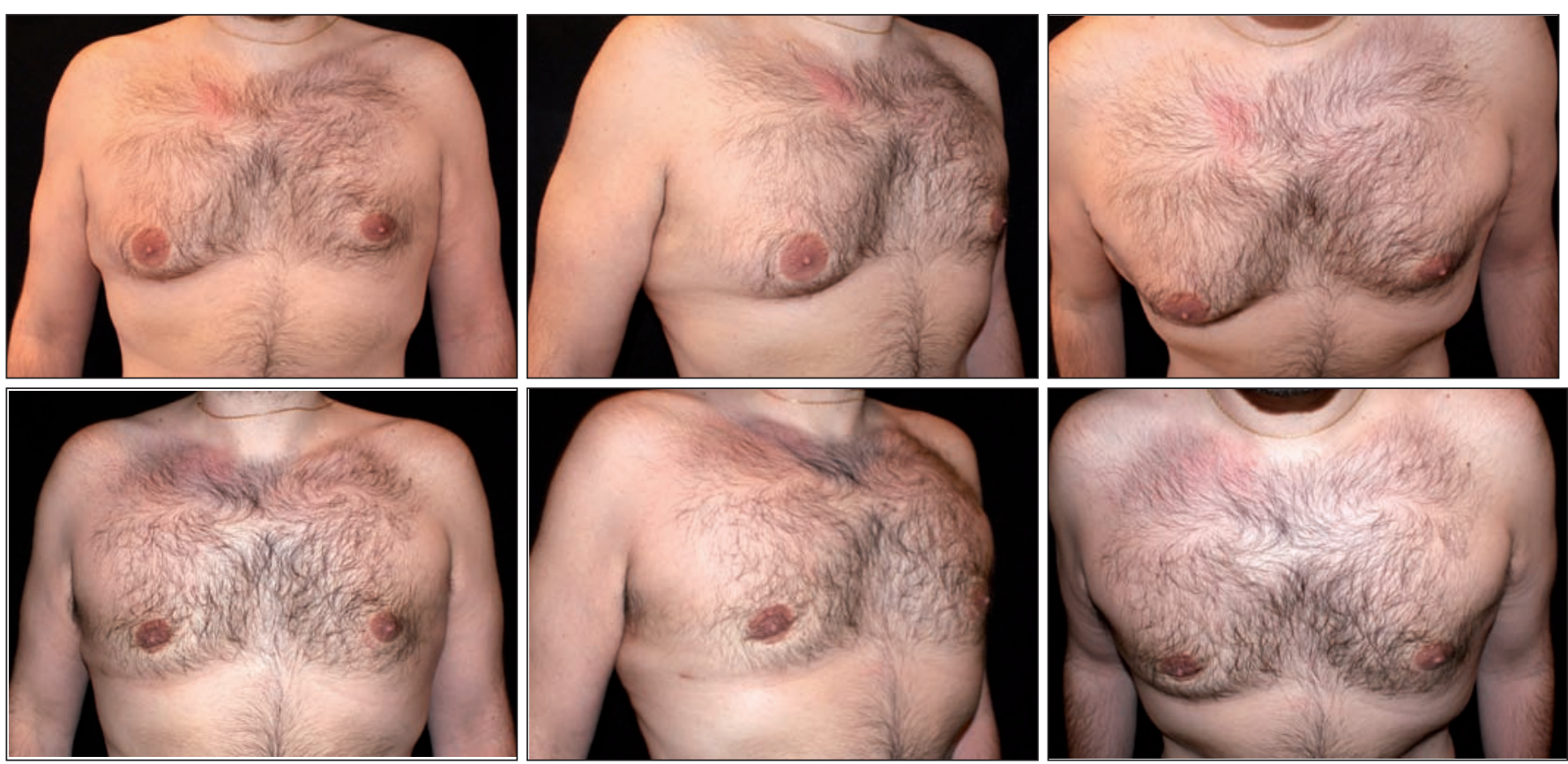

Fig. 13. Paciente de 38 años. Grupo 2 con asimetría (Ginecomastia adiposa y glandular solo del lado derecho). Desarrollo unilateral sin atipias al estudio histopatológico. Liposucción asistida por ultrasonido bilateral y resección directa de mama derecha por vía periareolar infeior. Postoperatorio al año.

El ultrasonido quirúrgico en liposucción fue utilizado por primera vez por Kloehn, quien describe para el mismo una alta selectividad por las células grasas disminuyendo así el sangrado, el edema postoperatorio, un menor porcentaje de equimosis y de irregularidades en el contorno. ${ }^{(10)}$ El VASER ${ }^{\circledR}$ es un dispositivo de pretratamiento para la extracción de grasa basado en la emisión de energía ultrasónica pulsada o continua para emulsionar la grasa antes de su aspiración por medio de succión. Sus cánulas más finas y el uso de energía ultrasónica per- 
miten una mayor emulsión de la grasa, creando túneles, disminuyendo el tiempo operatorio, preservando vasos y nervios y causando menor dolor y hematomas en el postoperatorio. ${ }^{(1)}$ Se demostró que el ultrasonido a una potencia de $1 \mathrm{MHz}$ no es efectivo en la destrucción de las células grasas, en cambio si lo es cuando se utiliza a 2 y $3 \mathrm{MHz}{ }^{(4)}$

En comparación con la liposucción tradicional, en la que no se utiliza este tipo de dispositivos como pretratamiento, Wong demostró que la USAL presenta un porcentaje de conversión a cirugía abierta y revisión postoperatoria del 25 frente al $39 \%$ y del 2 frente al $19 \%$ respectivamente, ${ }^{(9)}$ evidenciando una gran diferencia entre las dos técnicas ya que las cicatrices amplias y la necesidad de reintervención son indeseables para todo paciente. Además de tener buenos resultados estéticos percibidos por el cirujano, ${ }^{(8)}$ Masoumi reporta un $80 \%$ de satisfacción de los pacientes en un estudio donde recoge 609 casos ${ }^{(12)}$ muy similar a los resultados obtenidos en nuestro estudio donde la percepción de mejoría y satisfacción con el resultado final fue positiva. Otras ventajas perceptibles de esta técnica incluyen la corta curva de aprendizaje para su uso y una baja tasa de complicaciones, ${ }^{(13)}$ encontrando similitud en nuestros resultados con solo 2 casos de equimosis y sin presencia de hematomas, seromas, isquemia, necrosis ni sufrimiento cutáneo.

Gracias a nuestra experiencia previa mediante la cual obtuvimos una adecuada evolución de los pacientes tratados con USAL en otras zonas corporales ${ }^{(4)}$ (abdomen, cuello, flancos, brazos y miembros inferiores), en quienes encontramos mejor retracción cutánea, menor dolor y rápida recuperación, decidimos implementar el mismo tratamiento para los pacientes con ginecomastia. Los datos recopilados sugieren que dado el menor dolor postoperatorio, la no necesidad de drenajes y la baja incidencia de complicaciones, los pacientes tratados con USAL pueden tener un más rápido reintegro a las actividades cotidianas

Es por todo esto que creemos que, en manos experimentadas, la utilización del ultrasonido quirúrgico puede considerarse una herramienta segura para el paciente siempre y cuando se tomen las medidas locales necesarias para eliminar la posibilidad de quemaduras o de sufrimiento cutáneo.

\section{Conclusiones}

En nuestra experiencia, podemos concluir que la USAL en la región pectoral para el tratamiento de la ginecomastia, como en otras zonas del cuerpo, es una herramienta de importante utilidad como coadyuvante de la liposucción. Teniendo las adecuadas precauciones locales y técnicas, presenta baja tasa de complicaciones, mejora el contorno regional a simple vista, disminuye el dolor postoperatorio y logra una rápida reintegración de los pacientes a las actividades de su vida cotidiana.

\section{Dirección del autor}

Dr. Ernesto Moretti

Servicio de Cirugía Plástica

Hospital Privado Rosario

Roca 2440

(2000) Rosario, Argentina

Correo electrónico: contacto@esteticacentrum.com.ar

Bibliografía

1. Coiffman F. Cirugía Plástica, Reconstructiva Y Estética. 3rd ed. Venezuela: Amolca; 2006: Pp.3104-3106.

2. Simon BE, Hoffman S, Khan S. Classification and surgical correction of gynecomastia. Plast Reconstr Surg. 1973;51(1):48-52.

3. Shermak M. Body Contouring - Plastic Surgery Atlas, McGraw-Hill Ed. Baltimore, 2011; 4, Pp. 101-144.

4. Moretti E, Londoño L, Eid Caballero Y, Galetto M, Godoy A. Efecto del ultrasonido quirúrgico de alta potencia en el tejido dérmico y adiposo. Estudio experimental para aplicación en marcación abdominal. Cir plast Iiberolatinoam. 2016;42(3): 227-232

5. Johnson RE, Murad MH. Gynecomastia: Pathophysiology, Evaluation, and Management. Mayo Clinic Proceedings. 2009;84(11):1010-1015.

6. Krasopoulos G, Dusmet M, Ladas G, Goldstraw P: Nuss procedure improves the quality of life in young male adults with Pectus excavatum deformity. European Journal of Cardiothoracic Surgery 2006; 29: 1-5.

7. Petty PM, Et al. Gynecomastia: evolving paradigm of management and comparison of techniques. Plast Reconstr Surg. 2010;125(5):1301-1308.

8. Trelles M, et al. Lipolisis láser y liposucción en ginecomastia: retracción cutánea eficaz y segura. Rev. Col. Bras. Cir. 2013; 40(1):23-31.

9. Wong KY, Malata CM. Conventional versus ultrasound-assisted liposuction in gynaecomastia surgery: 13year review. J Plast Reconstr Aesthet Surg. 2014; 67(7):921-926.

10. Zoccali G, Et al. Multifrequency ultrasound-assisted liposuction: 5 years of experience. Aesthet Plast Surg. 2012;36(5):1052-1061.

11. Garcia O Jr, Nathan N. Comparative analysis of blood loss in suction-assisted lipoplasty and third-generation internal ultrasound-assisted lipoplasty. Aesthet Surg J. 2008;28(4):430-435.

12. Masoumi Lari, Et al. Determinants of patient satisfaction with ultrasound.assisted liposuction. Aesthet Surg J. 2010;30(5):714-719.

13. Hodgson EL, Et al. Ultrasonic liposuction in the treatment of gynecomastia. Plast Reconstr Surg. 2005; 116(2):646-653. 